ARTICLE

Received 8 Sep 2014 | Accepted 18 Feb 2015 | Published 14 Apr $2015 \quad$ DOl: 10.1038/ncomms7661

\title{
Polarization control at spin-driven ferroelectric domain walls
}

\author{
Naëmi Leo ${ }^{1}$, Anders Bergman ${ }^{2}$, Andres Cano ${ }^{3}$, Narayan Poudel ${ }^{4}$, Bernd Lorenz $^{4}$, Manfred Fiebig $^{1} \&$ Dennis Meier ${ }^{1}$
}

Unusual electronic states arise at ferroelectric domain walls due to the local symmetry reduction, strain gradients and electrostatics. This particularly applies to improper ferroelectrics, where the polarization is induced by a structural or magnetic order parameter. Because of the subordinate nature of the polarization, the rigid mechanical and electrostatic boundary conditions that constrain domain walls in proper ferroics are lifted. Here we show that spin-driven ferroelectricity promotes the emergence of charged domain walls. This provides new degrees of flexibility for controlling domain-wall charges in a deterministic and reversible process. We create and position a domain wall by an electric field in $\mathrm{Mn}_{0.95} \mathrm{CO}_{0.05} \mathrm{WO}_{4}$. With a magnetic field we then rotate the polarization and convert neutral into charged domain walls, while its magnetic properties peg the wall to its location. Using atomistic Landau-Lifshitz-Gilbert simulations we quantify the polarization changes across the two wall types and highlight their general occurrence.

\footnotetext{
${ }^{1}$ Department of Materials, ETH Zürich, Vladimir-Prelog-Weg 4, CH-8093 Zurich, Switzerland. ${ }^{2}$ Department of Physics and Astronomy, Uppsala University, PO Box 516, 75120 Uppsala, Sweden. ${ }^{3}$ CNRS, University of Bordeaux, ICMCB, UPR 9048, F-33600 Pessac, France. ${ }^{4}$ Department of Physics and Texas Center for Superconductivity, University of Houston, 3201 Cullen Boulevard, Houston, Texas 77204, USA. Correspondence and requests for materials should be addressed to D.M. (email: dennis.meier@mat.ethz.ch).
} 
F erroelectric domain walls represent a naturally occurring type of oxide interface that develops versatile electronic ground states. In particular, the polarity mismatch at charged walls leads to characteristics that clearly distinguish them from the interior of the domains they separate ${ }^{1-4}$. Such ferroelectric domain walls can behave as a two-dimensional insulator $^{5}$, become metallic ${ }^{6,7}$, show orientation-dependent electrical conductance ${ }^{8-10}$ or anisotropic magnetoresistance ${ }^{11}$, even when the bulk material has none of these properties. Exotic domain-wall phenomena are observed in archetypal ferroelectrics, such as $\mathrm{BaTiO}_{3}$ (refs 12,13), $\mathrm{PbZr}_{0.2} \mathrm{Ti}_{0.8} \mathrm{O}_{3}$ (refs 7,14,15) and $\mathrm{LiNbO}_{3}$ (ref. 16), where metastable charged domain walls can be induced by, for example, electric fields ${ }^{17}$. Additional degrees of freedom arise in so-called improper ferroelectrics, where the polarization emerges due to the coupling to a primary structural or magnetic order parameter. Here the boundary conditions imposed by the primary order parameter dominate so that unusual ferroelectric domain-wall configurations are readily stabilized even in the as-grown state. In the hexagonal manganites, for instance, both positively and negatively charged domain walls occur in the improper ferroelectric phase $\mathrm{s}^{9,18}$. Besides promoting the emergence of charged ferroelectric domain walls, the coupling to the primary order parameter provides a promising but so far unexplored handle to act on the local polarization by means other than electric fields, such as mechanical stress or magnetic fields.

In our work, we demonstrate the generation, positioning and subsequent polarization control at a ferroelectric domain wall using the spin-driven ferroelectric $\mathrm{Mn}_{0.95} \mathrm{Co}_{0.05} \mathrm{WO}_{4}$ (refs 19,20). A moderate voltage is applied to inject and place a $180^{\circ}$ ferroelectric domain wall with the spontaneous polarization $\mathbf{P}$ parallel to the wall (side-by-side configuration, $\uparrow \downarrow$ ). By applying a magnetic field we then continuously change the direction of $\mathbf{P}$ with respect to the spin-stabilized ferroelectric wall and thus transfer it gradually from a side-by-side state into head-to-head $(\rightarrow \leftarrow)$ or tail-to-tail $(\leftarrow \rightarrow)$ configurations. Atomistic LandauLifshitz-Gilbert (LLG) simulations are applied to analyse the impact of this transformation on the magnetic and electric domain-wall nanostructure. Our results show that the polarization state at spin-driven ferroelectric domain walls can readily be manipulated by applying magnetic fields. By moving the polarization with respect to the walls, but not the walls with a

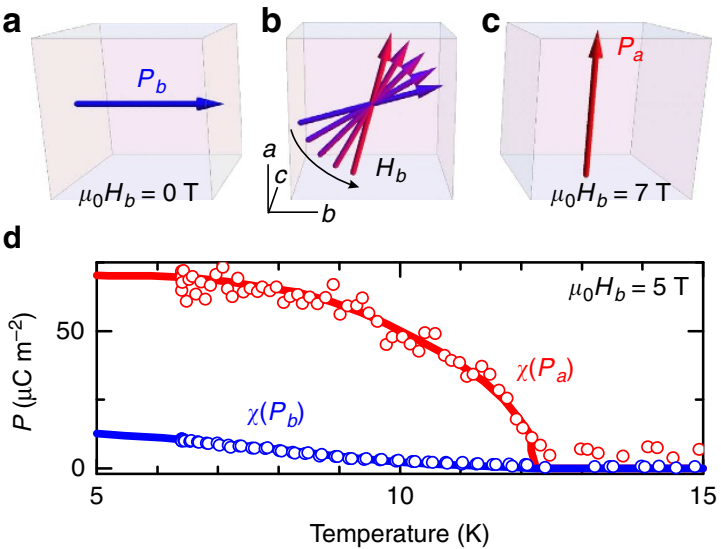

respect to the polarization, we demonstrate a new route for modifying the charge configuration of ferroelectric domain walls dynamically in a fully reversible process.

\section{Results}

Spin-stabilized ferroelectric domain walls. Spin-driven ferroelectrics display pronounced magnetoelectric coupling effects that allow for electric polarization control by applied magnetic fields ${ }^{21}$. This rigid correlation is expressed by the relation

$$
\mathbf{P} \sim \mathbf{e}_{i j} \times\left(\mathbf{S}_{i} \times \mathbf{S}_{j}\right),
$$

which links the electric polarization $\mathbf{P}$ to the vector chirality $\mathbf{S}_{i} \times \mathbf{S}_{j}$ of the underlying spin structure ${ }^{22,23}$ ( $i$ and $j$ denote the positions of neighbouring spin $\mathbf{S}, \mathbf{e}_{i j}$ is the unit vector connecting them). Such correlations are well understood as bulk phenomena $^{24}$ but they have not been utilized yet to manipulate the ferroelectric domain-wall states in a controlled way. Among the magnetically induced ferroelectrics $\mathrm{Mn}_{0.95} \mathrm{Co}_{0.05} \mathrm{WO}_{4}$ is outstanding because its electric bulk polarization $\mathbf{P}\left(T_{\mathrm{C}}=12 \mathrm{~K}\right)$ can be rotated continuously by about $90^{\circ}$ as illustrated in Fig. 1a-c (ref. 20). In the ground state, $\mathbf{P}$ is pointing along the crystallographic $b$ axis (Fig. 1a), but it gradually rotates towards the $a$ axis when a magnetic field $H \| b$ is applied (Fig. 1b). For $\mu_{0} H_{\mathrm{b}} \gtrsim 7 \mathrm{~T}$ a state with $\mathbf{P} \| a$ is stabilized (Fig. 1c). The continuous rotation is remarkably different from the magnetic field-driven first-order flop observed in other spin-spiral systems ${ }^{25,26}$. This gives the opportunity to smoothly modify the polarization configuration of ferroelectric domain walls.

To demonstrate the potential of spin-driven ferroelectricity for reversible configuration control at domain walls, we thus chose $\mathrm{Mn}_{0.95} \mathrm{Co}_{0.05} \mathrm{WO}_{4}$. The ferroelectric order of the material was probed by second harmonic generation (SHG), that is, frequency doubling of a light wave in a material (Methods). SHG is a particularly powerful tool for detecting polar phases because, in the leading order, it occurs only in non-centrosymmetric media, and thus emerges background-free when the inversion symmetry is broken by the ferroelectric order. SHG allows for visualizing and tracking the position of associated domain walls with a resolution of about $1 \mu \mathrm{m}$ (ref. 27). In Fig. 1d we show representative SHG signals at $\mu_{0} H_{b}=5 \mathrm{~T}$. The two contributions,
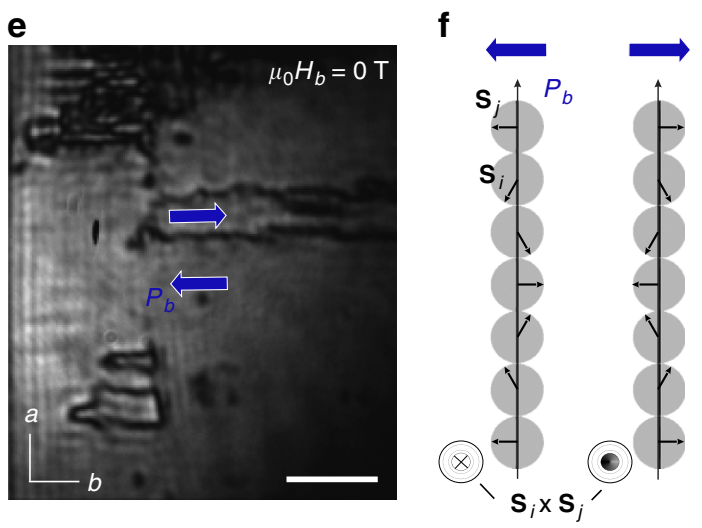

Figure 1 | Ferroelectric order and domains in $\mathbf{M n}_{\mathbf{0 . 9 5}} \mathbf{C o}_{\mathbf{0 . 0 5}} \mathbf{W O}_{\mathbf{4}}$. (a-c) Schematic illustrating the magnetic-field-induced polarization rotation from the $b$ axis at $0 \mathrm{~T}$ to the $a$ axis at $7 \mathrm{~T}$. (d) Temperature dependence of the polarization contributions $P_{b}$ and $P_{a}$ at 5 T measured by pyroelectric current (solid lines) and SHG (open circles), the latter at a photon energy of $2.2 \mathrm{eV}$. SHG intensities are scaled with respect to the pyroelectric current data $\left(I_{\mathrm{SHG}} \sim P^{2}\right)$. The correspondence of the data sets confirms that the SHG susceptibility is proportional to the ferroelectric polarization. (e) Ferroelectric domain structure imaged at $5.5 \mathrm{~K}$ after zero-field cooling. The ferroelectric domain walls are visible as dark lines. Side-by-side walls parallel to $P_{b}$ prevail in comparison to head-to-head and tail-to-tail walls perpendicular to $P_{b}$. Scale bar, $100 \mu \mathrm{m}$. (f) Spin structure in the ferroelectric domains. The direction of the spontaneous polarization is directly linked to the chirality of the spin spiral, which is described by the vector product of neighbouring spins $\mathbf{S}_{\mathrm{i}}$ and $\mathbf{S}_{\mathrm{j}}$. 
labelled $\chi\left(P_{a}\right)$ and $\chi\left(P_{b}\right)$, are proportional to the spontaneous polarization components along the $a$ and $b$ axis, respectively, in $\mathbf{P}=\left(P_{a}, P_{b}, 0\right)$ as indicated by the direct correspondence between SHG amplitude (open circles) and pyroelectric current data (solid lines).

We then exploited these SHG signals to image the ferroelectric domain structure. Figure 1e displays the distribution of ferroelectric domain walls in $\mathrm{Mn}_{0.95} \mathrm{Co}_{0.05} \mathrm{WO}_{4}$ imaged at $5 \mathrm{~K}$ after zero-field cooling. The SHG image is taken with light from $\chi\left(P_{b}\right)$ and reflects the position of ferroelectric $180^{\circ}$ domain walls by dark lines. These lines arise because the sign reversal of $P_{b}$ across the domain walls is converted into a $180^{\circ}$ phase difference of the SHG light field so that the SHG waves emitted from $+P_{b}$ and $-P_{b}$ domains interfere destructively and cancel at the domain-wall position. Figure 1e reveals that different types of ferroelectric $180^{\circ}$ domain walls are present. We note that every ferroelectric domain wall is also a magnetic domain wall across which the spin system changes its chirality as expressed by equation (1) and Fig. If.

The formation of ferroelectric side-by-side walls, that is, domain walls oriented parallel to the spontaneous polarization, as indicated by the arrows, is clearly preferred. Yet, sections with head-to-head and tail-to-tail walls perpendicular to $\mathbf{P}$ are also present. This distinguishes $\mathrm{Mn}_{0.95} \mathrm{Co}_{0.05} \mathrm{WO}_{4}$ from conventional ferroelectrics were the formation of such head-to-head and tailto-tail ferroelectric walls is generally avoided due to the energy cost associated to long-range dipole-dipole interactions. In $\mathrm{Mn}_{0.95} \mathrm{Co}_{0.05} \mathrm{WO}_{4}$, however, the presence of these walls reveals that these interactions are overruled by the energy gain associated to the underlying primary magnetic order so that electric dipoledipole interactions become secondary.

Magnetic field-driven change of the ferroelectric domain-wall configuration. In the next step we generated and positioned a single ferroelectric domain wall by applying the following poling procedure. First, we prepared our sample into a single-domain state by electric field cooling $(E \| b)$, which was achieved in a setup with external electrodes as sketched in Fig. 2a. The corresponding SHG image taken at $9 \mathrm{~K}$ with light from $\chi\left(P_{b}\right)$ is displayed in Fig. 2b and shows a homogeneous brightness level as expected for a uniformly polarized sample. We then applied a field of $4.8 \mathrm{kV} \mathrm{cm}^{-1}$ in order to create a domain wall and set its position. Figures $2 \mathrm{c}-\mathrm{f}$ document the domain-wall injection and subsequent sideways creep under the applied electric field. The image sequence shows that, after the injection, a net movement of the entire domain wall perpendicular to $\mathbf{P}$ occurs rather than nucleation and growth of a multitude of reverse domains. Just as in Fig. 1e the system thus displays a preference for ferroelectric side-by-side domain walls. Local head-to-head and tail-to-tail sections up to a length of hundred micrometres appear occasionally but disappear upon the continuing propagation of the wall (Fig. 2e). Once the domain wall had reached the centre of the sample (Fig. 2f) the electric field was switched off. Henceforth, the primary magnetic order stabilizes it against further motion.

To tune the polarization configuration of the injected and stabilized domain wall we then applied a magnetic field along the $b$ axis of the system and continuously rotated the ferroelectric polarization from $\mathbf{P} \| b$ towards $\mathbf{P} \| a$. Figure 3 a shows integrated SHG measurements (open circles) taken at $5 \mathrm{~K}$ while increasing the magnetic field $H_{b}$. In agreement with our pyroelectric current measurements (solid lines) the SHG data reveal that $\chi\left(P_{b}\right)$ decreases with increasing $H_{b}$, whereas $\chi\left(P_{a}\right)$ increases towards higher magnetic fields, reflecting the aforementioned polarization rotation ${ }^{20}$. In Fig. $3 b, c$ we present the corresponding spatially resolved SHG image for $\mathbf{P} \| b$ and $\mathbf{P} \| a$, that is, at $\mu_{0} H_{b}=0$ and a
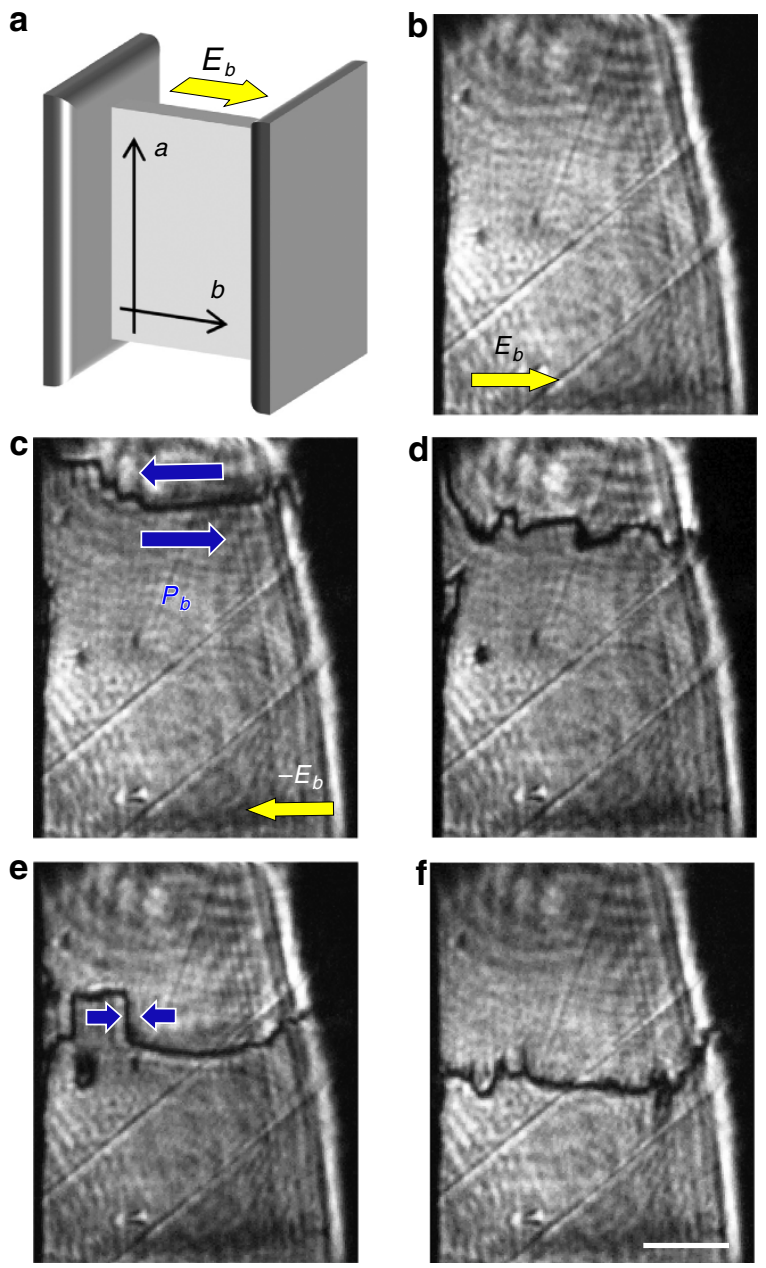

Figure 2 | Electric-field-driven injection and positioning of a domain wall. (a) Schematic illustration of the setup and sample orientation in between two metallic electrode plates. (b) SHG image of a ferroelectric singledomain state $\left(-P_{b}\right)$ taken after electric field poling. (c-f) Image sequence documenting the injection and subsequent positioning of a ferroelectric domain wall at a constant electric field $-E_{b}=4.8 \mathrm{kV} \mathrm{cm}^{-1}$. Note the general preference for side-by-side domain walls with only the transient occurrence of short head-to-head or tail-to-tail sections in the course of wall progression. All images were taken at $9 \mathrm{~K}$ at a SHG photon energy of $2.1 \mathrm{eV}$. Scale bar, $250 \mu \mathrm{m}$.

$6 \mathrm{~T}$, respectively. Both images show identical domain walls on the length scale resolved in our experiment. This demonstrates in a striking way that the domain wall is stable and unaffected by the continuous rotation of $\mathbf{P}$ in the magnetic field. Due to its spatial stability, the ferroelectric domain-wall state in $\mathrm{Mn}_{0.95} \mathrm{Co}_{0.05} \mathrm{WO}_{4}$ can readily be transformed from a side-by-side into a head-tohead or tail-to-tail configuration as sketched in Fig. 3b,c.

Microscopic domain-wall structure. To develop a microscopic domain-wall model and elaborate the impact of the magnetic field-driven configuration change, we performed atomistic simulations based on the LLG equation. We modelled the spindriven ferroelectric state with Heisenberg exchange interactions, magnetocrystalline anisotropy and Dzyaloshinskii-Moryia interactions as input parameters (Methods) ${ }^{28,29}$. The resulting electric polarization was calculated according to the KatsuraNagaosa-Balatzky model ${ }^{22}$ assuming that electric dipole-dipole interactions can be neglected as mentioned before. Our 

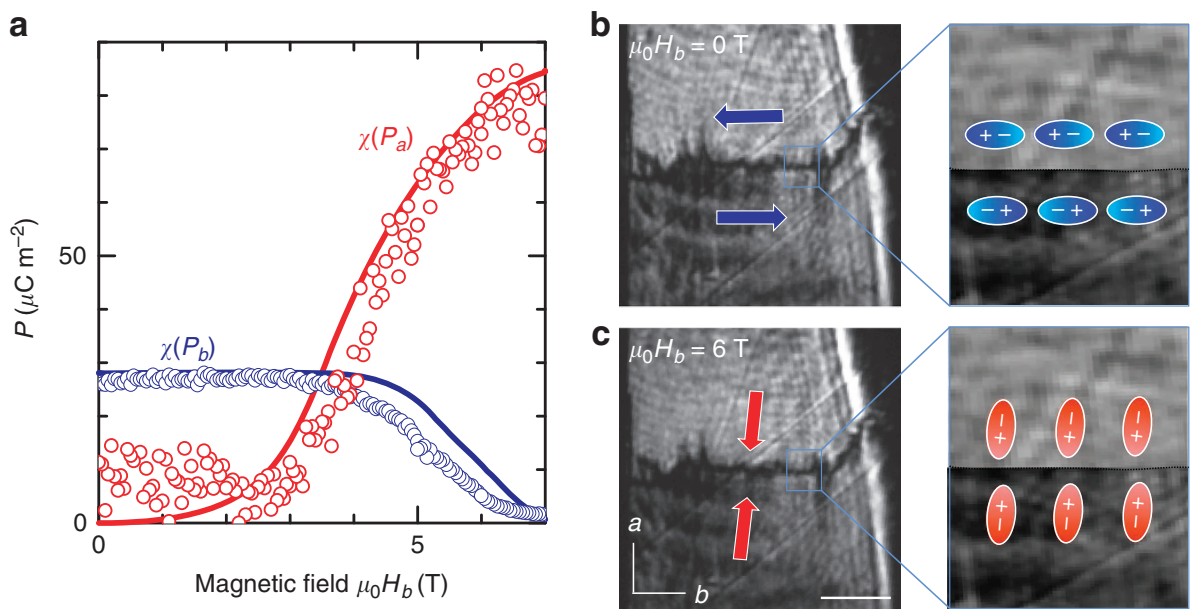

Figure 3 | Magnetic field control of the ferroelectric domain-wall state. (a) Pyroelectric current (solid lines) and integrated SHG measurements at $5 \mathrm{~K}$ tracking the magnetic-field-induced polarization rotation in $\mathrm{Mn}_{0.95} \mathrm{Co}_{0.05} \mathrm{WO}_{4}$. SHG intensities are normalized with respect to the pyroelectric current data $\left(I_{S H G} \sim P^{2}\right)$. (b) Spatially resolved SHG image of the injected and positioned ferroelectric domain wall (Fig. 2f) at zero magnetic field with $\mathbf{P} \| \boldsymbol{b}$. Blue arrows highlight the side-by-side configuration of the wall. The panel on the right illustrates the arrangement of electric dipoles at the domain wall. (c) SHG image taken after increasing $\mu_{0} H_{b}$ to $6 \mathrm{~T}$ with $\mathbf{P} \| a$ as indicated by the red arrows. All images were taken at $5 \mathrm{~K}$ at a SHG photon energy of $2.1 \mathrm{eV}$. The arrangement of the electric dipole moments at the wall is sketched on the right hand side. Scale bar, $250 \mu \mathrm{m}$.
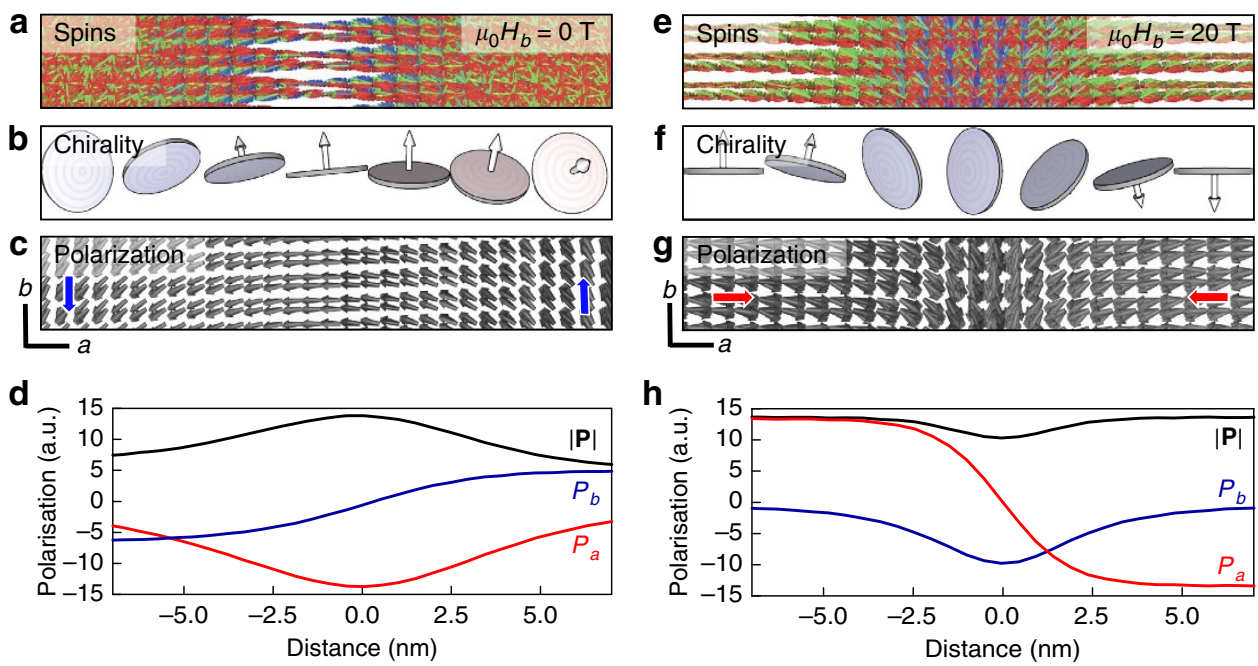

Figure 4 | Magnetic and ferroelectric domain-wall structure. (a) Simulation of the spin structure at a ferroelectric side-by-side wall at $\mu_{0} H_{b}=0 \mathrm{~T}$ with coloured arrows indicating individual spins $\mathbf{S}_{\mathrm{i}}$. (b) Evolution of the vector chirality $\mathbf{S}_{\mathrm{i}} \times \mathbf{S}_{\mathrm{j}}$ across the side-by-side wall. The discs represent the plane defined by $\mathbf{S}_{\mathrm{i}}$ and $\mathbf{S}_{\mathrm{j}}$ and arrows indicate the orientation of $\mathbf{S}_{\mathrm{i}} \times \mathbf{S}_{\mathrm{j}}$. (c) Calculated local ferroelectric polarization based on the spin structure in $\mathbf{a}$. Colored arrows show the polarization direction in adjacent domains. Grey arrows show the local polarization. (d) Changes in $|\mathbf{P}|$ and the components $P_{a}$ and $P_{b}$ across a side-by-side domain wall. (e-h) Like (a-d) but for $\mu_{0} H_{b}=20 \mathrm{~T}$.

simulations lead to a ferroelectric ground state $(T=0 \mathrm{~K})$ with $\mathbf{P} \| b$ and a ferroelectric transition temperature $T_{\mathrm{C}}=15 \mathrm{~K}$, which is in reasonable agreement with the experimental data. Domain walls were obtained by simulating a hot (disordered) system at $T \gg T_{\mathrm{N}}$ that was then cooled into the ordered state at $T=0.1 \mathrm{~K}$.

The results of our atomic LLG simulations for the case of a ferroelectric side-by-side domain wall at zero magnetic field $\left(\mu_{0} H_{b}=0 \mathrm{~T}\right)$ and for the associated magnetic-field-driven head-tohead state $\left(\mu_{0} H_{b}=20 \mathrm{~T}\right)$ are summarized in Fig. 4 . In Fig. $4 \mathrm{a}-\mathrm{c}$, we present the calculated spin structure, the vector chirality $S_{i} \times S_{j}$ and the corresponding polarization for the side-by-side domain wall. The simulation results visualize how the spin structure and the induced polarization change across side-by-side domain walls. Moreover, the projection of $\mathbf{P}$ shown in Fig. $4 \mathrm{c}$ reveals that the side-by-side domain wall in our system is not a simple Ising wall but has a more complex structure with a Néel (longitudinal) component such that $\mathbf{P}$ rotates in the $a b$-plane ${ }^{30,31}$. The evolution of the components $P_{a}$ and $P_{b}$ as well as the absolute value $|\mathbf{P}|$ of the polarization across the domain wall is displayed in Fig. $4 \mathrm{~d}$. The plot shows that the width of the spin-driven ferroelectric $180^{\circ}$ domain wall is about $10 \mathrm{~nm}$, which is comparable to the values found in proper ferroelectrics such as $\mathrm{PbZr}_{0.2} \mathrm{Ti}_{0.8} \mathrm{O}_{3}$ (ref. 15). For the induced head-to-head domain-wall state promoted by the magnetic field (Fig. 4e-h), the Néel component is preserved but with the smaller domain-wall width of about $5 \mathrm{~nm}$.

Remarkably, the simulations reveal that anomalous polarization values emerge at the centre of the domain walls. For the sideby-side domain wall, the polarization rotates from the $b$ to the $a$ axis at the wall centre, where it is about three times larger than in 
the bulk. This relation is in agreement with the bulk behaviour shown in Fig. 3a, where $P_{b}(0 \mathrm{~T})<P_{a}(7 \mathrm{~T})$. It highlights the ability of domain walls to locally enhance the spontaneous polarization $^{32}$. For the head-to-head wall $\mathbf{P}$ is suppressed compared with the bulk, yet with a finite value $P_{b}$ at the wall centre. Thus, we conclude that the magnetoelectric anisotropy, which is responsible for the different magnitude of $P_{b}$ and $P_{a}$ in the bulk, also determines the enhancement or suppression of $\mathbf{P}$ at the ferroelectric domain walls. Such variations in $\mathbf{P}$ may further be enhanced by the anisotropic dielectric response of the system $\left(\varepsilon_{a}>\varepsilon_{b}\right)$, which is not taken into account in our LLG simulations.

We note that the Ising-Néel walls obtained in our simulations are qualitatively different from those forming in proper ferroelectrics due to the flexoelectric effect. In the latter, the Néel component varies as an odd function across the domain wall so that $\mathbf{P}=0$ at the wall centre ${ }^{31,33}$. In our case we have $\mathbf{P} \neq 0$ across the entire cross-section of the domain wall, which reflects the multiferroic nature of our system. Similar to Néel components obtained via the flexoelectric effect, the extra components of $\mathbf{P}$ at the domain walls in $\mathrm{Mn}_{0.95} \mathrm{Co}_{0.05} \mathrm{WO}_{4}$ can reach values of about $100 \mu \mathrm{Cm}^{-2}$. In addition, these domain walls can get charged with a density of bound charges of up to $10^{5} \mathrm{C} \mathrm{m}^{-3}$, because there is a genuine Néel component that gives $\partial_{a} P_{a}=-\rho_{\text {bound }}<0$ across the wall. This implies that the transformation from a sideby-side wall, across which $\partial_{a} P_{a}$ changes sign so that the net charge is zero, into a head-to-head configuration changes the density of bound domain-wall charges.

\section{Discussion}

We expect that the magnetic-field-induced polarization rotation and the associated change in the domain-wall charge will affect the local density of mobile carriers (holes ${ }^{34}$ ) and hence the transport properties, analogous to the case of improper ferroelectric $\mathrm{ErMnO}_{3}$ (ref. 9). In $\mathrm{Mn}_{0.95} \mathrm{Co}_{0.05} \mathrm{WO}_{4}$, however, the low value of the spontaneous polarization and the cryogenic range of the multiferroic phase forfeit the technological merit. Since the bulk polarization of $\mathrm{Mn}_{0.95} \mathrm{Co}_{0.05} \mathrm{WO}_{4}$ is in the order of $50 \mu \mathrm{C} \mathrm{m}^{-2}$, the amount of charge to screen head-to-head and tail-to-tail walls is about three orders of magnitude smaller than, for example, in the hexagonal manganites ${ }^{9}$. Thus, domain-wall conductance with currents in the fA range is expected, which is challenging to detect in local transport measurements at lowtemperature. Successful and ongoing attempts at obtaining spindriven ferroelectrics with a spontaneous polarization of $>1,000 \mu \mathrm{C} \mathrm{m}^{-2}$ and higher ordering temperatures are, however, continuously improving these odds ${ }^{35-37}$.

Another important issue is the time dependence of the orderparameter reorientation. Phenomenologically it has been demonstrated that a reorientation by acting on the improper (electric) order parameter tends to be slow with reversal times in the millisecond range ${ }^{38,39}$. By acting on the primary (magnetic) order parameter, theory predicts scenarios with a reversal within a few picoseconds, yet at so far unrealistically large fields ${ }^{40}$. Experimentally, all-optical magnetic switching in 1-100 ps has been demonstrated, yet in non-multiferroic compounds and with the theory for this process still being under development ${ }^{41,42}$.

In conclusion, we demonstrated the continuous polarization control at a ferroelectric domain wall in the spin-driven ferroelectric $\mathrm{Mn}_{0.95} \mathrm{Co}_{0.05} \mathrm{WO}_{4}$. The spontaneous polarization at the domain wall was continuously rotated by application of a magnetic field, which smoothly transferred the wall between a neutral side-by-side and a nominally charged head-to-head state in a fully reversible process. The primary magnetic order inducing the ferroelectric polarization protects the wall against any reorientation that may be favoured by this charging process.
The electric and magnetic structure of the domain walls throughout the polarization-reorientation procedure was analysed by LLG simulations. By moving the polarization with respect to the walls instead of the walls with respect to the polarization, we point out a route for the reversible modification of the electric polarization configuration at ferroelectric domain walls. With this approach we add an additional degree of flexibility for dynamical domain-wall engineering. Even though the results obtained on $\mathrm{Mn}_{0.95} \mathrm{Co}_{0.05} \mathrm{WO}_{4}$ are conceptual rather than device oriented, the continuing and successful search for multiferroics with larger spontaneous polarization and ordering temperature turns the design of domain-wall-based devices for nanoelectronics into a realistic goal.

\section{Methods}

Second harmonic generation. The emission of light at frequency $2 \omega$ from a material irradiated with light at frequency $\omega$ is described by the equation $\mathbf{S}(2 \omega)=\varepsilon_{0} \chi \mathbf{E}(\omega) \mathbf{E}(\omega)$. Here $\mathbf{E}(\omega)$ denotes the electric field components of the incident light, $\mathbf{S}(2 \omega)$ is the induced nonlinear polarization acting as source for the emitted SHG wave, and the nonlinear susceptibility tensor $\chi$ characterizes the symmetry of the host material. By temperature- and polarization-dependent SHG spectroscopy in a transmission geometry, we identified the nonzero SHG susceptibilities $\chi_{a b b}, \chi_{b a a}$ and $\chi_{b b b}$. In agreement with the symmetry analysis and with pyroelectric current measurements, these couple linearly to the components $P_{a}$ or $P_{b}$ of the ferroelectric polarization. In a simplified notation, the susceptibilities $\chi_{a b b}$

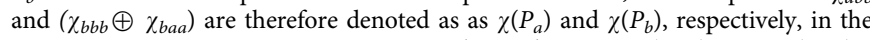
main text. Maximum SHG emission was obtained at $2.2 \mathrm{eV}\left(\chi_{a b b}\right), 2.1 \mathrm{eV}\left(\chi_{b a a}\right)$, and $2.2 \mathrm{eV}\left(\chi_{b b b}\right)$ so that these photon energies were employed in the experiments.

LLG simulations. To parameterize the spin Hamiltonian, Heisenberg exchange interactions, magnetocrystalline anisotropy and Dzyaloshinskii-Moryia interactions served as input ${ }^{43}$. The Heisenberg exchange and Dzyaloshinskii-Moryia interactions were at first calculated for undoped $\mathrm{MnWO}_{4}$ using density functiona theory calculations based on the multiple scattering theory and a local density approximation. The calculated exchange interactions led to a spin-spiral ground state at $0 \mathrm{~K}$ with the wave vector $\mathbf{k}=(0.17,0.5,0.41)$. To approximate the larger magnetic anisotropy of $\mathrm{Mn}_{0.95} \mathrm{Co}_{0.05} \mathrm{WO}_{4}$ in comparison with $\mathrm{MnWO}_{4}$, we considered two kinds of anisotropies, a 'double-easy-axis' anisotropy ${ }^{44}$ and an easy-plane anisotropy with the plane chosen such that the two easy axes are within the easy plane. All the simulations presented in the main text were performed using a system of $200 \times 24 \times 24$ unit cells.

\section{References}

1. Salje, E. K. H. Multiferroic domain boundaries as active memory devices: trajectories towards domain boundary engineering. Chem. Phys. Chem. 11, 940-950 (2010).

2. Salje, E. K. H. \& Zhang, H. Domain boundary engineering. Phase Transit. 82, 452-469 (2009).

3. Catalan, G., Seidel, J., Ramesh, R. \& Scott, J. F. Domain wall nanoelectronics. Rev. Mod. Phys. 84, 119-156 (2012).

4. Gureev, M. Y., Tagantsev, A. K. \& Setter, N. Head-to-head and tail-to-tail domain walls in an isolated ferroelectric. Phys. Rev. B 83, 184104 (2011).

5. Choi, T. et al. Insulating interlocked ferroelectric and structural antiphase domain walls in multiferroic $\mathrm{YMnO}_{3}$. Nat. Mater. 9, 253-258 (2010).

6. Seidel, J. et al. Conduction at domain walls in oxide multiferroics. Nat. Mater. 8, 229-234 (2009).

7. Maksymovych, P. et al. Tunable metallic conductance in ferroelectric nanodomains. Nano Lett. 12, 209-213 (2012).

8. Eliseev, E. A., Morozovska, A. N., Svechnikov, G. S., Gopalan, V. \& Shur, V. Y. Static conductivity of charged domain walls in uniaxial ferroelectric semiconductors. Phys. Rev. B 83, 235313 (2011).

9. Meier, D. et al. Anisotropic conductance at improper ferroelectric domain walls. Nat. Mater. 11, 284-288 (2012).

10. Vasudevan, R. et al. Domain wall geometry controls conduction in ferroelectrics. Nano Lett. 12, 5524-5531 (2012).

11. Lee, J. H. et al. Spintronic functionality of $\mathrm{BiFeO}_{3}$ domain walls. Adv. Mater. 26, 7078-7082 (2014).

12. Sluka, T., Tagantsev, A. K., Bednyakov, P. \& Setter, N. Free-electron gas at charged domain walls in insulating $\mathrm{BaTiO}_{3}$. Nat. Commun. 4, 1808 (2013).

13. Sluka, T., Tagantsev, A. K., Damjanovic, D., Gureev, M. \& Setter, N. Enhanced electromechanical response of ferroelectrics due to charged domain walls. Nat. Commun. 3, 748 (2012).

14. Guyonnet, J., Gaponenko, I., Gariglio, S. \& Paruch, P. Conduction at domain walls in insulating $\mathrm{Pb}\left(\mathrm{Zr}_{0.2} \mathrm{Ti}_{0.8}\right) \mathrm{O}_{3}$ thin films. Adv. Mater. 23, 5377-5382 (2011). 
15. Jia, C.-L. et al. Atomic-scale study of electric dipoles near charged and uncharged domain walls in ferroelectric films. Nat. Mater. 7, 57-61 (2008).

16. Schröder, M. et al. Conducting domain walls in lithium niobate single crystals. Adv. Func. Mater. 22, 3936-3944 (2012).

17. Whyte, J. R. et al. Ferroelectric domain wall injection. Adv. Mater. 26, 293-298 (2014).

18. Wu, W., Horibe, Y., Lee, N., Cheong, S.-W. \& Guest, J. R. Conduction of topologically protected charged ferroelectric domain walls. Phys. Rev. Lett. 108, 077203 (2012).

19. Liang, K.-C. et al. The complex multiferroic phase diagram of $\mathrm{Mn}_{1-\mathrm{x}} \mathrm{Co}_{\mathrm{x}} \mathrm{WO}_{4}$. New J. Phys. 14, 073028 (2012).

20. Liang, K.-C. et al. Field-induced continuous rotation of the polarization in multiferroic $\mathrm{Mn}_{0.95} \mathrm{Co}_{0.05} \mathrm{WO}_{4}$. J. Appl. Phys. 111, 07D903 (2012).

21. Kimura, T. Spiral magnets as magnetoelectrics. Annu. Rev. Mater. Res. 37, 387-413 (2007).

22. Katsura, H., Nagaosa, N. \& Balatsky, A. Spin currents and magnetoelectric effect in noncollinear magnets. Phys. Rev. Lett. 95, 057205 (2005).

23. Mostovoy, M. Ferroelectricity in spiral magnets. Phys. Rev. Lett. 96, 067601 (2006).

24. Tokura, Y. \& Seki, S. Multiferroics with spiral spin order. Adv. Mater. 22, 1554-1565 (2010).

25. Taniguchi, K., Abe, N., Takenobu, T., Iwasa, Y. \& Arima, T. Ferroelectric polarization flop in a frustrated magnet $\mathrm{MnWO}_{4}$ induced by a magnetic field. Phys. Rev. Lett. 97, 097203 (2006).

26. Kimura, T. et al. Magnetic control of ferroelectric polarization. Nature 426, 55-58 (2003).

27. Fiebig, M., Pavlov, V. V. \& Pisarev, R. V. Second-harmonic generation as a tool for studying electronic and magnetic structures of crystals. J. Opt. Soc. Am. B 22, 96-118 (2005).

28. Ebert, H. et al. The Munich SPR-KKR package, version 6.3. Available at http://ebert.cup.uni-muenchen.de/SPRKKR (2010).

29. Ebert, H., Kodderitzsch, D. \& Minar, J. Calculating condensed matter properties using the KKR-Green's function method-recent developments and applications. Rep. Prog. Phys. 74, 096501 (2011).

30. Houchmandzadeh, B., Lajzerowicz, J. \& Salje, E. K. H. Order parameter coupling and chirality of domain walls. J. Phys. Cond. Matter 3, 5163-5169 (1991).

31. Yudin, P. V., Tagantsev, A. K., Eliseev, E. A., Morozovska, A. N. \& Setter, N. Bichiral structure of ferroelectric domain wall driven by flexoelectricity. Phys. Rev. B 86, 134102 (2012).

32. Van Aert, S. et al. Direct observation of ferrielectricity at ferroelastic domain boundaries in $\mathrm{CaTiO}_{3}$ by electron microscopy. Adv. Mater. 24, 523-527 (2012).

33. $\mathrm{Gu}, \mathrm{Y}$. et al. Flexoelectricity and ferroelectric domain wall structures: Phase-field modeling and DFT calculations. Phys. Rev. B 89, 174111 (2014).

34. Bharati, R. \& Singh, R. A. Electrical conduction in manganese tungstate. J. Phys. Chem. Sol. 7, 641-644 (1982).

35. Kitagawa, Y. et al. Low-field magnetoelectric effect at room temperature. Nat. Mater. 9, 797-802 (2010).
36. Rocquefelte, X., Schwarz, K., Blaha, P., Kumar, S. \& van den Brink, J. Room-temperature spin-spiral multiferroicity in high-pressure cupric oxide. Nat. Commun. 4, 2511 (2013).

37. Johnson, R. et al. Giant improper ferroelectricity in the ferroaxial magnet $\mathrm{CaMn}_{7} \mathrm{O}_{12}$. Phys. Rev. Lett. 108, 067201 (2012).

38. Baum, M. et al. Kinetics of the multiferroic switching in $\mathrm{MnWO}_{4}$. Phys. Rev. B 89, 144406 (2014).

39. Hoffmann, T., Thielen, P., Becker, P., Bohatý, L. \& Fiebig, M. Time-resolved imaging of magnetoelectric switching in multiferroic $\mathrm{MnWO}_{4}$. Phys. Rev. B 84, 184404 (2011).

40. Mochizuki, M. \& Nagaosa, N. Theoretically predicted picosecond optical switching of spin chirality in multiferroics. Phys. Rev. Lett. 105, 147202 (2010).

41. Vahaplar, K. et al. Ultrafast path for optical magnetization reversal via a strongly nonequilibrium state. Phys. Rev. Lett. 103, 117201 (2009).

42. Radu, I. et al. Transient ferromagnetic-like state mediating ultrafast reversal of antiferromagnetically coupled spins. Nature 472, 205-208 (2010).

43. Skubic, B. et al. A method for atomistic spin dynamics simulations: Implementation and examples. J. Phys. Condens. Matter 20, 315203 (2008).

44. Hollmann, N. et al. Local symmetry and magnetic anisotropy in multiferroic $\mathrm{MnWO}_{4}$ and antiferromagnetic $\mathrm{CoWO}_{4}$ studied by soft X-ray absorption spectroscopy. Phys. Rev. B 82, 184429 (2010).

\section{Acknowledgements}

We thank T. Hoffmann and M. Trassin for helpful discussions. The work at the ETH Zürich was supported by the SNSF projects 200021_149192 and 200021_147080. A.B. acknowledges the Swedish Research Council (VR) and eSSENCE for the financial support. The simulations were performed on resources provided by the Swedish National Infrastructure for Computing (SNIC) at the National Supercomputer Centre (NSC). The work at Houston was supported in part by the US Air Force Office of Scientific Research, the T.L.L. Temple Foundation, the John J. and Rebecca Moores Endowment, and the State of Texas through TCSUH.

\section{Author contributions}

N.L. performed and analysed the SHG measurements, A.B. performed the LLG simulations, A.C. contributed to the discussion and analysis, and N.P. and B.L. provided the samples and conducted the pyroelectric and magnetoelectric current measurements. D.M. initiated this project, coordinated the work and, together with M.F., supervised the experiments. All authors discussed the results and commented on the manuscript.

\section{Additional information}

Competing financial interests: The authors declare no competing financial interests.

Reprints and permission information is available at http://npg.nature.com/ reprintsandpermissions/

How to cite this article: Leo, N. et al. Polarization control at spin-driven ferroelectric domain walls. Nat. Commun. 6:6661 doi: 10.1038/ncomms7661 (2015). 